\title{
ANÁLISE DO CONTRASTE DA PENALIDADE E DA RECOMPENSA (PRC): IDENTIFICANDO OPORTUNIDADES DE MELHORIA EM UM SERVIÇO
}

\section{PENALTY AND REWARD CONTRAST ANALYSIS (PRC): IDENTIFYING IMPROVEMENT OPPORTUNITIES IN A SERVICE}

\section{JAIME DAGOSTIM PICOLO}

Mestre em Administração pela Universidade Regional de Blumenau (FURB). Professor da Universidade do Extremo Sul Catarinense (Unesc). Avenida Universitária, 1105, Universitário - Criciúma - SC - CEP 88806-000 E-mail: jaime@unesc.net

\section{GERSON TONTIN}

Doutor em Engenharia Mecânica pela Universidade Federal de Santa Catarina (UFSC). Professor do Doutorado em Contabilidade e Administração da Universidade Regional de Blumenau (FURB). Rua Paraguai, 436, ap. 101, Centro - Blumenau - SC - CEP 89050-020 E-mail: tontini@furb.br 


\section{RESUMO}

As organizações estão inseridas em um ambiente competitivo no qual a melhoria contínua dos seus produtos auxilia a manter ou incrementar novos negócios. Nesse processo, é relevante ouvir o cliente, pois é ele quem decide se o produto ou serviço será utilizado ou não, sendo então essencial conhecer os atributos do produto que intensificam o nível de satisfação dos clientes. Este artigo tem como objetivo apresentar o método de análise do contraste da penalidade e da recompensa (Penalty and Reward Contrast analysis - PRC), que identifica atributos relevantes na influência da satisfação do consumidor. Para ilustrar o método PRC, realizou-se uma pesquisa avaliando $\mathrm{I} 6$ atributos de supermercados, com uma amostra de 290 respondentes. $\mathrm{O}$ resultado da pesquisa revela que a análise do contraste da penalidade e da recompensa apresenta os atributos que influenciam a satisfação geral, mas descarta atributos que os consumidores não têm experiência de utilização, entre eles, os inovadores.

\section{PALAVRAS-CHAVE}

Satisfação do consumidor; Serviço ao cliente; Análise de oportunidades de melhoria; Importância de atributos; Método PRC.

\section{ABSTRACT}

The organizations are inserted in a competitive environment where continuous improvement of their products aids to keep or increase new businesses. In this process, it is relevant to hear the customer because it is him who decides if the product or service will be used or no, being then essential to know the attributes of the product that intensifies the level of customers' satisfaction. This article has as objective to present the PRC - Penalty and Reward Contrast analysis - to identify the relevant attributes that influence consumer's satisfaction. To illustrate the PRC method, the paper evaluated I6 attributes of supermarkets, with a sample 
of 290 respondents. The result of the research reveals that the PRC identifies the attributes that influence the general satisfaction, but it discards attributes that the consumers don't have experience, among them, the innovations.

\section{KEYWORDS}

Consumer's satisfaction; Customer service; Analysis of improvement opportunities; Importance of attributes; Penalty and reward contrast analysis.

\section{INTRODUÇÃO}

Em decorrência da crescente competição entre as organizações para incrementar e manter mercados, a satisfação dos clientes tornou-se um dos fatores relevantes para o sucesso comercial de médio e longo prazo; porém, manter clientes satisfeitos pode não ser suficiente. Segundo Kotler (2000), clientes meramente satisfeitos mudam de fornecedor quando descobrem uma oferta melhor. É necessário oferecer bens e serviços que deixem os clientes mais que satisfeitos.

Anderson e Mittal (2000), estudando empresas que fazem parte do Barômetro de Satisfação do Consumidor Sueco, verificaram que um acréscimo de I\% em média no índice de satisfação de clientes está associado a 2,37\% de incremento no retorno sobre o investimento. Clientes muito satisfeitos ou encantados são menos motivados a se ocuparem de pesquisas com outros fornecedores, repetindo o consumo com a empresa que os deixou encantados. Por outro lado, Anderson e Mittal (2000) encontraram que um decréscimo de I\% na satisfação está associado com 5,08\% de redução do retorno sobre o investimento. Em um mercado competitivo, os clientes abandonam determinado produto ou fornecedor quando insatisfeitos, substituindo-o por outra opção que lhes traga maior satisfação. Além disso, eles tendem a divulgar suas experiências desagradáveis, afastando outros consumidores. Isso sugere que o decréscimo em satisfação é aproximadamente duas vezes mais prejudicial do que o benefício com o acréscimo equivalente.

Conforme Juran (I992), a satisfação do cliente é alcançada quando o desempenho dos atributos do produto atende às suas necessidades. Uma avaliação competitiva do desempenho dos atributos dos produtos é essencial no desenvolvimento e melhoria de bens e serviços, uma vez que os clientes também efetuam essa avaliação quando decidem que produtos irão comprar. 
Métodos diversos têm sido utilizados para direcionar os esforços de melhoria em bens e serviços. Um deles é a matriz de importância e desempenho, que recomenda ações de melhoria, manutenção ou desinvestimento com base no valor de importância e desempenho de cada atributo (MARTILLA; JAMES, I977). Em geral, dados provenientes de pesquisas sobre satisfação do consumidor são utilizados para construir uma matriz bi-dimensional, em que a importância é mostrada pelo eixo y e o desempenho do atributo pelo eixo x. A matriz é dividida em quatro quadrantes. $\mathrm{O}$ atributo que tiver alta importância e alto desempenho representa uma possível vantagem competitiva. Nesse caso, a empresa deve manter o bom trabalho. $\mathrm{O}$ atributo que tiver alta importância, mas baixo desempenho, deverá receber atenção imediata. Para aumentar a satisfação geral, dirigentes de empresas deveriam se concentrar em melhorá-lo. Atributos com baixa importância e baixo desempenho não necessitam de esforço adicional. Atributos com alto desempenho, porém com baixa importância, podem indicar que a empresa esteja desperdiçando recursos que poderiam ser mais bem aproveitados em outras aplicações.

Segundo Garver (2003), o desempenho pode ser obtido pela avaliação da satisfação dos clientes com os atributos. Já a importância dos atributos pode ser obtida pela importância declarada ou importância derivada estatisticamente. A declarada é obtida por uma nota dada pelo cliente para cada atributo, em uma escala que pode variar de sem importância a extremamente importante. A derivada estatisticamente pode ser obtida por uma equação de regressão linear entre a satisfação com os atributos individuais (variáveis independentes) e a satisfação geral com o produto ou serviço (variável dependente). Nesse caso a importância de cada atributo é dada pelo valor dos coeficientes ( $\beta$ ) da equação, e o atributo que obtiver o maior valor de $\beta$ é considerado o mais importante, pois proporciona maior impacto na satisfação geral.

A matriz de importância e desempenho tem como pressuposto a independência entre o desempenho e a importância. Porém existem evidências de que a importância se altera em função da satisfação. Matzler et al. (2004), analisando a importância derivada estatisticamente para atributos com alto ou baixo desempenho, demonstram que ela pode variar com o desempenho. Já a importância declarada, como demonstrada por Picolo (2005), apresenta correlação positiva com o módulo da satisfação com os atributos.

A linearidade entre o desempenho e a satisfação é um outro pressuposto dessa matriz, porque considera que qualquer atributo, ao ser melhorado, refletirá em aumento proporcional de satisfação. Contudo, alguns tipos de atributos não proporcionam aumento na satisfação do consumidor na mesma proporção que o aumento de desempenho, apresentando uma relação não-linear entre desempenho e satisfação geral. A falta de independência entre importância e desempenho e a relação não-linear entre satisfação com os atributos individuais e satisfação 
geral pode levar a decisões equivocadas de melhoria quando utilizada a matriz de importância e desempenho (HUISKONEN; PIRTTILÄ, I998; TONTINI; SILVEIRA, 2005).

O modelo Kano de qualidade atrativa e obrigatória (KANO et al., I984) identifica a relação não-linear entre desempenho e satisfação dos atributos, classificando-os como obrigatórios, unidimensionais, atrativos, neutros ou reversos. Kano et al. (I984), Berger et al. (I993) e Matzler et al. (I996) sugerem que a classificação dos atributos pode ser identificada perguntando-se aos consumidores sobre sua reação, ou satisfação, quando os atributos têm desempenho superior (questão positiva) e inferior (questão negativa). Dependendo das respostas para a questão positiva e negativa, o atributo pode ser identificado como atrativo, obrigatório, unidimensional, neutro ou reverso. Porém, de acordo com Tontini e Silveira (2005), no método tradicional de classificação segundo o modelo Kano, quando o consumidor responde seu grau de satisfação em relação a cada atributo está pensando sobre o atributo individual e não sobre o serviço como um todo. A melhoria de um atributo pode causar aumento na satisfação com aquele atributo, contudo seu efeito na satisfação geral pode ser pequeno devido à interação dos diferentes atributos entre si, não sendo possível identificar se os atributos vão de fato influenciar a satisfação geral.

A análise do contraste da penalidade e da recompensa (PRC) identifica as relações lineares e não-lineares entre satisfação com os atributos e satisfação geral. Com essa identificação e o diagnóstico do desempenho atual dos atributos, a organização poderá priorizar investimentos em melhorias que influenciam na satisfação geral. Este trabalho tem como objetivo apresentar a análise do PRC contraste da penalidade e da recompensa, como alternativa à análise pela matriz de importância e desempenho. O artigo está estruturado da seguinte forma: a Seção 2 apresenta a metodologia usada para ilustrar o PRC; a Seção 3, a análise do PRC com variáveis intervalares e binárias; a Seção 4, a análise do PRC aplicada ao caso estudado neste trabalho; a Seção 5, as limitações do método; e a Seção 6 apresenta as conclusões do trabalho.

\section{MATERIAIS E MÉTODO}

Uma pesquisa com clientes de supermercados foi utilizada para ilustrar o método PRC. Embora qualquer produto ou serviço pudesse ser objeto de estudo, o serviço supermercados foi utilizado por ser de conhecimento geral dos respondentes. A pesquisa foi efetuada com uma amostra probabilística aleatória de 290 alunos da Universidade do Extremo Sul Catarinense (Unesc), clientes de supermercados da cidade de Criciúma, Santa Catarina. Como os alunos provêm de diferentes regiões e classes sociais e freqüentam supermercados diferentes, considerou-se 
essa amostra como adequada para a realização do trabalho. Para aumentar a confiabilidade da pesquisa, todos os respondentes aceitaram participar da pesquisa, tomando tempo suficiente para responder às questões formuladas. Os atributos selecionados para aplicação da pesquisa (Quadro I) foram definidos com o auxílio de um grupo de foco realizado com três gerentes de supermercados e dez consumidores.

Por meio de um questionário fechado, com uma escala variando de extremamente insatisfeito $(-5)$ a extremamente satisfeito $(+5)$, pediu-se que os respondentes avaliassem sua satisfação em relação ao desempenho atual dos atributos pesquisados e também a satisfação geral com o supermercado que mais freqüentam. Solicitou-se ainda que declarassem se o supermercado que mais freqüentam disponibiliza ou não os atributos ATı, AT2, AT3, AT4, AT5, AT6, AT7, AT9, ATı4 e ATı6 (ver descrição dos atributos no Quadro I), por esses serem dicotômicos e terem desempenho mensurado pela presença ou ausência.

\section{QUADRO I}

ATRIBUTOS SELECIONADOS PARA A PESQUISA

\begin{tabular}{llll}
\hline CÓD. & DESCRIÇÃO & CÓD. & DESCRIÇÃO \\
\hline AT1 & Proximidade do supermercado & AT9 & Cartão fidelidade com bônus progressivo \\
\hline AT2 & Compra por Internet & AT10 & Tamanho das filas no caixa \\
\hline AT3 & Lojas diversas anexas & AT11 & Qualidade das frutas, verduras e legumes \\
\hline AT4 & Local para deixar crianças & AT12 & Preços abaixo da média do mercado \\
\hline AT5 & Degustação de produtos & AT13 & Diversidade de marcas por produto \\
\hline AT6 & Música ambiente & AT14 & Câmeras de vigilância \\
\hline AT7 & Vagas suficientes no estacionamento & AT15 & Cortesia do atendimento \\
\hline AT8 & Limpeza e arrumação da loja & AT16 & Disponibilidade de sanitários para clientes \\
\hline
\end{tabular}

Fonte: Elaborado pelos autores.

A idade média dos respondentes é de 24 anos, sendo $39 \%$ do sexo masculino e 6r\% do sexo feminino. Declaram-se $76 \%$ solteiros, i9\% casados e $5 \%$ divorciados e viúvos. No quesito renda familiar, 31\% declaram receber até $\mathrm{R} \$$ I.000,00, 42,I\% de R $\$$ I.00I, 00 até $\mathrm{R} \$ 3.000,00,22,4 \%$ acima de $\mathrm{R} \$ 3.000,00$ e 4,5\% não declararam renda. Essas características da amostra assemelham-se às características da população de alunos da Unesc. Quanto à freqüência de utilização de supermercados, 92\% dos respondentes afirmam utilizar supermercados pelo menos uma vez ao mês, o que contribui para a qualidade das respostas 
devido ao uso constante do serviço. Os dois supermercados que foram mais citados serão identificados como SMA e SMB e os demais como OUT SM. O supermercado SMA foi citado por $30 \%$ da amostra, o SMB por $25 \%$ e os demais respondentes citaram outros supermercados.

\section{ANÁLISE dO CONTRASTE DA PENALIDADE E DA RECOMPENSA (PRC)}

A importância derivada estatisticamente, como comentado anteriormente, pode ser encontrada pela equação de regressão linear $Y=\beta_{O}+\beta_{I} X_{I}+\beta_{2} X_{2}+\ldots+$ $\beta n X n$, onde $Y$ representa a satisfação geral com o produto ou serviço e $X$, a satisfação com os atributos individuais. O pressuposto principal deste método é que existe uma relação linear entre satisfação com os atributos individuais e satisfação geral (MATZLER; SAUERWEIN, 2002). Apesar disso, existem evidências de que a relação entre essas variáveis nem sempre é linear. Mas qual seria o problema de considerar que todas as relações são lineares? E como se poderia diagnosticar a possível relação não-linear entre satisfação dos atributos e satisfação geral?

Observa-se no gráfico da Figura I à esquerda, a correlação entre satisfação com o atributo ATII - Qualidade das frutas, verduras e legumes e satisfação geral com os supermercados estudados neste trabalho.

\section{FIGURA I}

\section{EXEMPLO DE RELAÇÃO NÃO-LINEAR}

\section{E O AJUSTE DE REGRESSÃO LINEAR}

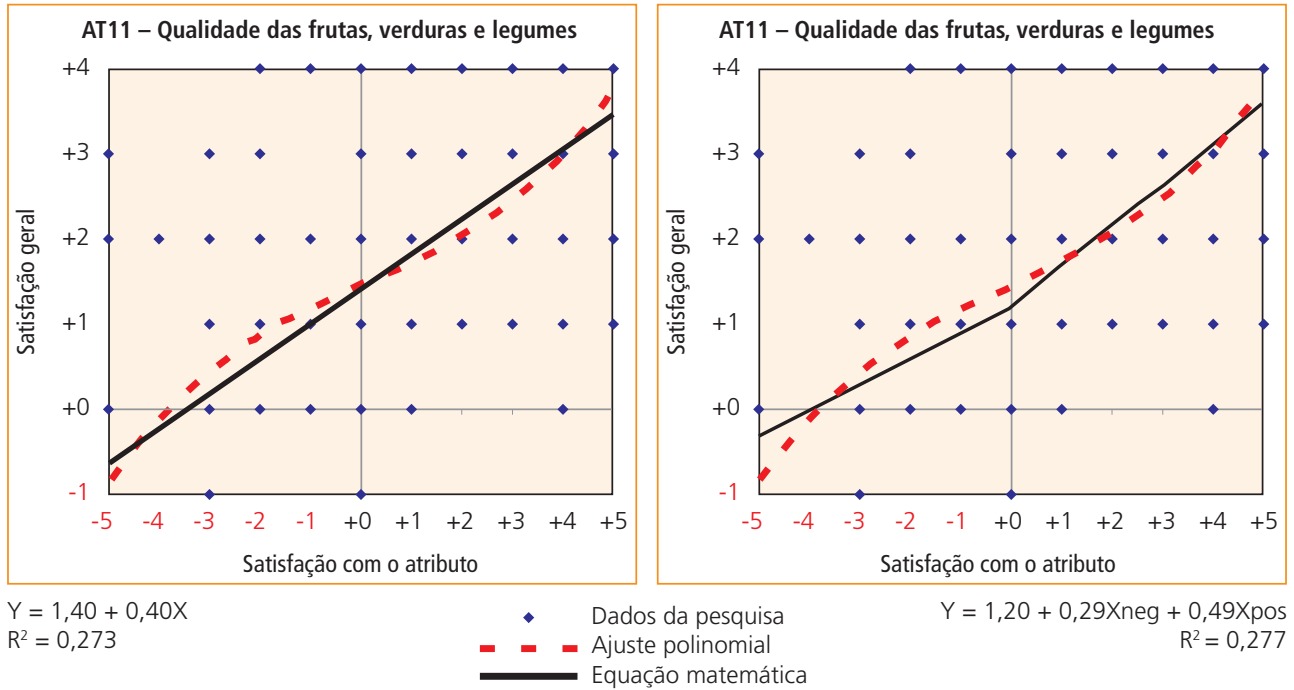

Fonte: Elaborada pelos autores. 
Se for efetuado um ajuste de regressão linear com o modelo $Y=\beta_{\mathrm{O}}+\beta_{\mathrm{I}} \mathrm{X}_{\mathrm{I}}$, a equação matemática que explica a satisfação geral pode ser estimada como $\mathrm{Y}$ $=\mathrm{I}, 40+0,40 \mathrm{X}$. Isso quer dizer que para cada unidade de alteração da variável independente X (satisfação com o atributo), a variável dependente $\mathrm{Y}$ (satisfação geral) se altera em 0,40 unidade.

A análise do contraste da penalidade e da recompensa (PRC) considera a possibilidade de que a relação entre satisfação do atributo e satisfação geral não seja linear, modelando a relação atributo versus satisfação geral com uma regressão múltipla com variáveis dummy ( $\mathrm{Y}=\beta \mathrm{O}+\beta_{\mathrm{I}} \mathrm{X}_{\mathrm{I}}$ neg $+\beta_{2} \mathrm{X}_{\mathrm{I}}$ pos) (ANDERSON; MITTAL, 2000; AUDRAIN, 2004; BRANDT, I987; CHEUNG; LEE, 2005; FUCHS; WEIERMAIR, 2004; MATZLER et al., 2004; MATZLER; SAUERWEIN, 2002; TING; CHEN, 2002). Nesse caso, a satisfação com o atributo individual é recodificada em duas variáveis dummy. Quando a satisfação com o atributo está acima de determinado valor de referência, a variável dummy de recompensa ( $\mathrm{X}_{\mathrm{I}}$ pos) é maior que zero e a variável dummy de penalidade ( $\mathrm{X}_{\mathrm{I}}$ neg) é igual a zero. Se a satisfação com o atributo for abaixo da referência, a variável dummy de penalidade $\left(\mathrm{X}_{\mathrm{I}}\right.$ neg) é menor que zero e $\mathrm{X}_{\mathrm{I}}$ pos é igual a zero. E quando a satisfação é igual à referência, $\mathrm{X}_{\mathrm{I}}$ pos e $\mathrm{X}_{\mathrm{I}}$ neg são iguais a zero. Desse modo, obtêm-se dois coeficientes de regressão para uma única variável independente: um coeficiente quando satisfação com o atributo for menor e outro, quando for maior do que o valor referencial determinado.

No gráfico da Figura I à direita esse valor de referência foi assumido como o (zero) para X (satisfação do atributo) na escala variando de -5 a +5. Assim, o modelo da satisfação geral foi estimado como Y = I,20 + 0,29Xneg + 0,49Xpos. Nesse caso, quando a satisfação com o atributo for positiva, para cada unidade de aumento ou diminuição na satisfação com o atributo, a satisfação geral se altera em o,49. Quando a satisfação com o atributo for negativa, para cada unidade de aumento ou diminuição na satisfação com o atributo, a satisfação geral se altera em o,29. Avaliando-se o $\mathrm{R}^{2}$ das duas equações mostradas na Figura I, verifica-se que a equação com variáveis dummy representa melhor a relação entre satisfação com o atributo ATıI Qualidade das frutas, verduras e legumes - e satisfação geral do que a equação de regressão linear simples. Caso a relação entre satisfação com o atributo e satisfação geral seja linear, os coeficientes de recompensa e penalidade serão semelhantes.

O exemplo mostrado na Figura I é um modelo monoatributo, em que se analisa a relação de apenas um atributo interferindo na satisfação geral. A análise do PRC pode também ser efetuada com um modelo multiatributos ( $\mathrm{Y}=\beta_{\mathrm{O}}$ $+\beta_{\mathrm{I}} \mathrm{X}_{\mathrm{I}}$ pos $+\beta_{2} \mathrm{X}_{2}$ neg $+\ldots+\beta_{\mathrm{n}} \mathrm{X}_{\mathrm{n}}$ pos $+\beta_{\mathrm{n}} \mathrm{X}_{\mathrm{n}}$ neg), onde se analisa simultaneamente a interferência de vários atributos na satisfação geral (MITTAL; ROSS; 
BALDASARE, I998). O modelo multiatributo é considerado mais adequado, pois, conforme Mittal, Ross e Baldasare (I998), o cliente pode estar satisfeito ou insatisfeito com aspectos diferentes do mesmo produto ou serviço. Em um restaurante, por exemplo, pode-se estar altamente satisfeito com a comida e descontente com o serviço prestado. Além disso, a análise de PRC com equações de regressão monoatributo pode deixar de identificar relações existentes entre os diversos atributos. No modelo multiatributo, as variáveis competem entre si para estabelecer a magnitude dos coeficientes, resultando em um modelo superior.

A interpretação dos resultados da análise de PRC dependerá de que ponto a recompensa é maior, igual ou menor à penalidade. Se a recompensa exceder em valor à penalidade, recomenda-se dirigir esforços para maximizar avaliações positivas de clientes no atributo em questão. No entanto, se a penalidade exceder o valor da recompensa, recomenda-se dirigir esforços para minimizar avaliações negativas dos clientes.

Apesar de os trabalhos citados usarem a análise de PRC para identificar o impacto dos atributos na satisfação geral, a maneira de codificar as variáveis dummy e a definição dos valores de referência tem sido efetuada de forma diversa na literatura. De modo geral, a codificação das variáveis dummy ocorre de duas maneiras: binária ou intervalar.

\subsection{ANÁLISE DE PRC COM VARIÁVEIS DUMMY BINÁRIAS}

Anderson e Mittal (2000), Audrain, (2004), Brandt (1987), Fuchs e Weiermair (2004), Matzler et al. (2004) e Matzler e Sauerwein (2002) utilizaram equações de regressão com variáveis dummy binárias para análise de PRC. A codificação das variáveis dummy binárias é feita da seguinte maneira: quando a avaliação do atributo estiver abaixo de determinada referência (por exemplo, a referência pode ser o se a escala de medição da satisfação for de $-5 a+5)$, atribui-se $\circ$ para a variável de recompensa e I para a de penalidade; quando a avaliação do atributo for acima da referência, atribui-se I para a variável de recompensa e o para a de penalidade; e se a avaliação do atributo for igual à referência, as variáveis de recompensa e de penalidade recebem valor o. Por isso, a recodificação pode ser chamada de dummy binária, visto que assume valor o ou I. Um exemplo dessa recodificação é mostrado na Tabela I. 


\section{TABELA I}

EXEMPLO DE RECODIFICAÇÃO DA SATISFAÇÃO DO ATRIBUTO COM VARIÁVEIS DUMMY BINÁRIAS

\begin{tabular}{cc|ccc}
\hline \multicolumn{2}{c|}{ DADOS DE PESQUISA } & VARIÁVEIS RECODIFICADAS COM VARIÁVEIS DUMMY BINÁRIAS \\
\hline $\begin{array}{c}\text { SATISFAÇÃO } \\
\text { COM O ATRIBUTO }\end{array}$ & $\begin{array}{c}\text { SATISFAÇÃO } \\
\text { GERAL }\end{array}$ & PENALIDADE & RECOMPENSA & SATISFAÇÃO GERAL \\
\hline-5 & 3 & 1 & 0 & 3 \\
\hline 4 & 4 & 0 & 1 & 4 \\
\hline 0 & 5 & 0 & 0 & 5 \\
\hline
\end{tabular}

Fonte: Elaborada pelos autores.

Conduzindo-se uma análise de regressão múltipla, onde as variáveis dummy são as variáveis independentes e a satisfação geral, a variável dependente da equação $\mathrm{Y}=\beta \mathrm{o}+\beta_{\mathrm{I}}$ recompensa $+\beta_{2}$ penalidade, obtém-se dois coeficientes de regressão, um para a recompensa e outro para a penalidade.

A Figura 2 apresenta o ajuste da equação de regressão do atributo ATII com variáveis dummy binárias ( $\mathrm{Y}=0,68+\mathrm{I}, 93$ recompensa $-0,60$ penalidade).

\section{FigurA 2}

\section{MODELO DE SATISFAÇÃO COM VARIÁVEIS DUMMY BINÁRIAS DO ATRIBUTO ATIII}

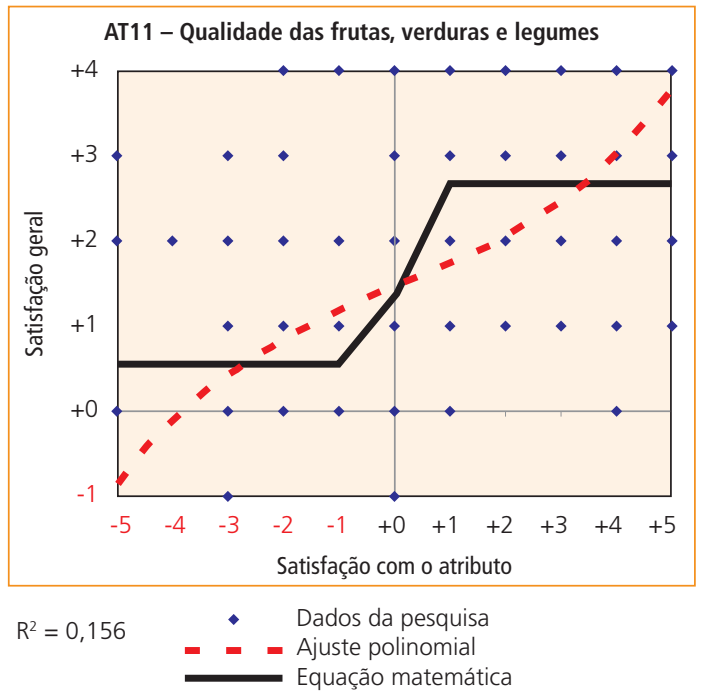

Fonte: Elaborada pelos autores. 
Nesse exemplo, quando a satisfação do atributo é positiva, a recompensa é igual a I, a penalidade é igual a $\circ$ (zero) e a satisfação geral é estimada em um resultado igual a $\mathrm{Y}=0,68+(\mathrm{I}, 93 \times \mathrm{I})-(0,60 \times 0)=2,6 \mathrm{I}$. O mesmo ocorre quando a satisfação do atributo é negativa. A recompensa é igual a 0 , a penalidade é igual a I e a satisfação geral é estimada em Y $=0,68+(\mathrm{I}, 93 \times 0)-(0,60 \times \mathrm{I})$ $=0,08$. Sendo assim, o coeficiente de recompensa representa o acréscimo de satisfação geral média quando a satisfação do atributo é positiva e o coeficiente de penalidade representa o decréscimo médio da satisfação geral quando a satisfação do atributo for negativa.

O Quadro 2 apresenta como os autores Anderson e Mittal (2000), Audrain, (2004), Brandt (1987), Fuchs e Weiermair (2004), Matzler et al. (2004) e Matzler e Sauerwein (2002) recodificaram as variáveis de avaliação dos atributos em penalidade e recompensa em suas pesquisas. Na terceira e quinta colunas, tem-se como as avaliações dos atributos foram transformadas na variável dummy de penalidade e recompensa. A quarta coluna apresenta em que condições ambas as variáveis (penalidade e recompensa) possuem valor zero.

\section{QUADRO 2}

RESUMO DA AVALIAÇÃO DOS ATRIBUTOS E SUA RECODIFICAÇÃO BINÁRIA

\begin{tabular}{|c|c|c|c|c|}
\hline AVALIAÇÃO DOS ATRIBUTOS & $\begin{array}{c}\text { AVALIAÇÃO GERAL } \\
\text { DO PRODUTO }\end{array}$ & $\begin{array}{l}\text { VARIÁVEL } \\
\text { PENALIDADE } \\
\text { TEM VALOR } \\
\text { IGUALA } 1\end{array}$ & $\begin{array}{l}\text { PENALIDADE E } \\
\text { RECOMPENSA } \\
\text { COM VALOR } \\
\text { IGUAL A ZERO }\end{array}$ & $\begin{array}{l}\text { VARIÁVEL } \\
\text { RECOMPENSA } \\
\text { TEM VALOR } \\
\text { IGUALA } 1\end{array}$ \\
\hline \multicolumn{5}{|l|}{$\begin{array}{l}\text { Brandt (1987) } \\
\text { significância 5\% }\end{array}$} \\
\hline $\begin{array}{l}\text { Escala categórica com rótulos } \\
\text { de Muito pior do que o } \\
\text { esperado, Um pouco pior que } \\
\text { o esperado, Igual ao esperado, } \\
\text { Um pouco melhor do que o } \\
\text { esperado e Muito melhor do } \\
\text { que o esperado }\end{array}$ & $\begin{array}{l}\text { A satisfação geral } \\
\text { foi avaliada com: } \\
\text { Muito satisfeito, } \\
\text { Um pouco satisfeito, } \\
\text { Neutro, Um pouco } \\
\text { insatisfeito e Muito } \\
\text { insatisfeito }\end{array}$ & $\begin{array}{l}\text { Muito pior } \\
\text { que e Um } \\
\text { pouco pior } \\
\text { que }\end{array}$ & & $\begin{array}{l}\text { Um pouco } \\
\text { melhor que } \\
\text { e Muito } \\
\text { melhor que }\end{array}$ \\
\hline \multicolumn{5}{|l|}{ Anderson e Mittal (2000) } \\
\hline $\begin{array}{l}\text { Escala de } 7 \text { pontos } \\
(1 \text { = Abaixo das expectativas, } \\
3=\text { Como esperado e } \\
7 \text { = Acima das expectativas) }\end{array}$ & $\begin{array}{l}\text { Escala de } 7 \text { pontos } \\
(1 \text { = Extremamente } \\
\text { insatisfeito, } \\
7=\text { Extremamente } \\
\text { satisfeito })\end{array}$ & $\begin{array}{l}\text { Abaixo das } \\
\text { expectativas }\end{array}$ & $\begin{array}{l}\text { Como } \\
\text { esperado }\end{array}$ & $\begin{array}{l}\text { Acima das } \\
\text { expectativas }\end{array}$ \\
\hline
\end{tabular}




\section{QUADRO 2 (CONTINUAÇÃO)}

\section{RESUMO DA AVALIAÇÃO DOS ATRIBUTOS E SUA RECODIFICAÇÃO BINÁRIA}

\begin{tabular}{|c|c|c|c|c|}
\hline AVALIAÇÃO DOS ATRIBUTOS & $\begin{array}{l}\text { AVALIAÇÃO GERAL } \\
\text { DO PRODUTO }\end{array}$ & $\begin{array}{l}\text { VARIÁVEL } \\
\text { PENALIDADE } \\
\text { TEM VALOR } \\
\text { IGUALA } 1\end{array}$ & $\begin{array}{c}\text { PENALIDADE E } \\
\text { RECOMPENSA } \\
\text { COM VALOR } \\
\text { IGUAL A ZERO }\end{array}$ & $\begin{array}{l}\text { VARIÁVEL } \\
\text { RECOMPENSA } \\
\text { TEM VALOR } \\
\text { IGUAL A } 1\end{array}$ \\
\hline \multicolumn{5}{|l|}{$\begin{array}{l}\text { Matzler e Sauerwein (2002) } \\
\text { significância } 5 \% \text { e } \mathrm{R}^{2} \mathrm{a}=0,489\end{array}$} \\
\hline $\begin{array}{l}\text { Avaliação dos atributos com: } \\
\text { Muito satisfeito, Um pouco } \\
\text { satisfeito, Neutro, Um pouco } \\
\text { insatisfeito, Muito insatisfeito }\end{array}$ & $\begin{array}{l}\text { Escala de Likert de } \\
5 \text { pontos de Muito } \\
\text { satisfeito a Muito } \\
\text { insatisfeito }\end{array}$ & $\begin{array}{l}\text { Um pouco } \\
\text { insatisfeito } \\
\text { e Muito } \\
\text { insatisfeito }\end{array}$ & $\begin{array}{l}\text { Um pouco } \\
\text { satisfeito e } \\
\text { neutro }\end{array}$ & $\begin{array}{l}\text { Muito } \\
\text { satisfeito }\end{array}$ \\
\hline \multicolumn{5}{|l|}{$\begin{array}{l}\text { Audrain }(2004) \\
\text { significância } 10 \%\end{array}$} \\
\hline \multicolumn{2}{|c|}{$\begin{array}{l}\text { Avaliou a satisfação com Escala de Likert bipolar } \\
\text { ancorada Com muito satisfeito e Muito insatisfeito }\end{array}$} & $\begin{array}{l}\text { Insatisfeito } \\
\text { e Muito } \\
\text { insatisfeito }\end{array}$ & Satisfeito & $\begin{array}{l}\text { Muito } \\
\text { satisfeito }\end{array}$ \\
\hline \multicolumn{5}{|l|}{$\begin{array}{l}\text { Fuchs e Weiermair (2004) } \\
\text { significância } 10 \% \text { e } R^{2}=0,377\end{array}$} \\
\hline \multicolumn{2}{|c|}{$\begin{array}{l}\text { Escala de Likert com } 5 \text { pontos (1- Não satisfeito com } \\
\text { nada e } 5 \text { - Altamente satisfeito) }\end{array}$} & $\begin{array}{l}\text { Avaliação } \\
1 \text { e } 2\end{array}$ & $\begin{array}{l}\text { Avaliação } \\
3 \text { e } 4\end{array}$ & $\begin{array}{l}\text { Avaliação } \\
5\end{array}$ \\
\hline \multicolumn{5}{|l|}{$\begin{array}{l}\text { Matzler et al. (2004) } \\
\text { significância } 10 \% \text { e } R^{2}=0,50\end{array}$} \\
\hline $\begin{array}{l}\text { Escala de } 1 \text { a } 10 \text {, medido o } \\
\text { desempenho de Extremamente } \\
\text { baixo a Extremamente alto }\end{array}$ & $\begin{array}{l}\text { Escala de } 1 \text { a } \\
\text { 10, medindo a } \\
\text { satisfação de } \\
\text { Extremamente baixa } \\
\text { a Extremamente alta }\end{array}$ & $\begin{array}{c}\text { Baixo } \\
\text { desempenho }\end{array}$ & $\begin{array}{c}\text { Médio } \\
\text { desempenho }\end{array}$ & $\begin{array}{c}\text { Alto } \\
\text { desempenho }\end{array}$ \\
\hline
\end{tabular}

Fonte: Elaborado pelos autores.

Apesar de os autores não declararem se o modelo de regressão utilizado em suas pesquisas foi ou não multiatributo, Matzler e Sauerwein (2002), Fuchs e Weiermair (2004) e Matzler et al. (2004) apresentaram apenas um valor de $\mathrm{R}^{2}$ (coeficiente de determinação). Isso permite deduzir que seus modelos são multiatributo $\left(Y=\beta_{\circ}+\beta_{I} X_{I}\right.$ pos $+\beta_{2} X_{2}$ neg $+\ldots+\beta_{n} X_{n}$ pos $+\beta_{n} X_{n}$ neg). Autores como Audrain, (2004), Fuchs e Weiermair (2004) e Matzler e Sauerwein (2002), por considerarem que, quando o cliente responde Satisfeito na escala de avaliação, está na verdade Neutro, recodificam a referência Zero para as variáveis 
de penalidade e de recompensa não no meio da escala, mas, sim, no ponto da escala que consideram neutro, segundo sua base teórica de análise.

Quanto à significância dos coeficientes de regressão, de um modo geral, as pesquisas dos autores citados no Quadro 2 apresentaram as magnitudes dos coeficientes de todos os atributos. Porém, para classificarem os atributos, Audrain (2004), Fuchs e Weiermair (2004) e Matzler et al. (2004) consideraram apenas magnitudes significativas ao nível de I0\%, enquanto Brandt (1987), Matzler e Sauerwein (2002) consideraram 5\%.

\subsection{ANÁLISE DE REGRESSÃO COM VARIÁVEIS DUMMY INTERVALARES}

Ting e Chen (2002) e Cheung e Lee (2005), ao efetuarem as recodificações de suas avaliações dos atributos em penalidade e recompensa, não utilizaram as variáveis dummy binárias (com valores o ou I), mas sim variáveis dummy intervalares, conforme descrito no Quadro 3.

\section{QUADRO 3}

RESUMO DA AVALIAÇÃO DOS ATRIBUTOS E SUA RECODIFICAÇÃO COM ESCALA INTERVALAR

\begin{tabular}{|c|c|c|c|c|}
\hline $\begin{array}{l}\text { AVALIAÇÃO DOS } \\
\text { ATRIBUTOS }\end{array}$ & $\begin{array}{l}\text { AVALIAÇÃO DO } \\
\text { PRODUTO }\end{array}$ & $\begin{array}{l}\text { PENALIDADE } \\
\text { COM ESCALA } \\
\text { INTERVALAR } \\
\text { NEGATIVA }\end{array}$ & $\begin{array}{l}\text { PENALIDADE E } \\
\text { RECOMPENSA } \\
\text { COM VALOR } \\
\text { IGUAL A ZERO }\end{array}$ & $\begin{array}{l}\text { RECOMPENSA } \\
\text { COM ESCALA } \\
\text { INTERVALAR } \\
\text { POSITIVA }\end{array}$ \\
\hline \multicolumn{5}{|l|}{$\begin{array}{l}\text { Ting e Chen (2002) } \\
\text { significância 5\% }\end{array}$} \\
\hline $\begin{array}{l}\text { Escala de Likert com } \\
8 \text { pontos (-4 Discordo } \\
\text { extremamente } \\
\text { e +4 Concordo } \\
\text { extremamente) }\end{array}$ & $\begin{array}{l}\text { Escala de } 10 \\
\text { pontos com } 1 \\
\text { (baixo grau de } \\
\text { satisfação) e } 10 \\
\text { (alto grau de } \\
\text { satisfação) }\end{array}$ & $\begin{array}{c}1,39 ; 1,10 ; \\
0,69 ; 0,00 \\
\text { (valor do } \\
\text { logaritmo } \\
\text { natural da } \\
\text { escala negativa) }\end{array}$ & $\begin{array}{c}\text { Centro } \\
\text { da escala }\end{array}$ & $\begin{array}{c}0,00 ; 0,69 ; 1,10 ; \\
1,39 \\
\text { (valor do } \\
\text { logaritmo } \\
\text { natural da escala } \\
\text { positiva) }\end{array}$ \\
\hline \multicolumn{5}{|c|}{$\begin{array}{l}\text { Cheung e Lee ( } 2005) \\
\text { significância } 10 \% \text { e } R^{2}=0,550\end{array}$} \\
\hline $\begin{array}{l}\text { Escala de Likert } \\
\text { com } 1 \text { Discordo } \\
\text { completamente } \\
\text { e } 7 \text { Concordo } \\
\text { completamente }\end{array}$ & $\begin{array}{l}\text { Escala de Likert } \\
\text { de Muito } \\
\text { insatisfeito a } \\
\text { Muito satisfeito }\end{array}$ & $\begin{array}{c}\text { Multiplicou por } \\
\text { uma constante } \\
\text { para que o } \\
\text { valor mínimo da } \\
\text { escala seja }=-7\end{array}$ & $\begin{array}{l}\text { Média da } \\
\text { avaliação }\end{array}$ & $\begin{array}{l}\text { Multiplicou por } \\
\text { uma constante } \\
\text { para que o valor } \\
\text { máximo da } \\
\text { escala seja }=+7\end{array}$ \\
\hline
\end{tabular}

Fonte: Elaborado pelos autores. 
Ting e Chen (2002) recodificaram a penalidade e a recompensa utilizando o logaritmo natural, com o valor de referência estabelecido no centro da escala. Já Cheung e Lee (2005) utilizaram como referência na recodificação da penalidade e da recompensa o valor médio de satisfação atual de cada atributo e recodificaram a escala acima e abaixo dessa referência. Um exemplo dessa recodificação é apresentado na Tabela 2, em que o valor médio de satisfação do atributo é igual a I. Nesse caso é criada uma tabela de variáveis recodificadas, na qual a satisfação geral é repetida com os mesmos valores e cada avaliação da satisfação do atributo é transformada em penalidade e recompensa. Toda avaliação é comparada com o valor médio de satisfação do atributo. Se a avaliação for maior do que a média, a recompensa será igual à avaliação menos a média $(4-\mathrm{I}=3)$ e a penalidade será igual a zero. Se a avaliação for menor do que a média, a penalidade será igual à avaliação menos a média $(-2-\mathrm{I}=-3)$ e a recompensa será igual a zero. Se a avaliação for igual à média, ambas, penalidade e recompensa, serão iguais a zero. A penalidade e a recompensa indicam a distância entre a avaliação do atributo e a satisfação média atual.

\section{TABELA 2}

EXEMPLO DE RECODIFICAÇÃO DA SATISFAÇÃO DO ATRIBUTO COM VARIÁVEIS DUMMY INTERVALARES COM REFERÊNCIA IGUAL A SATISFAÇÃO ATUAL

\begin{tabular}{cc|ccc}
\hline \multicolumn{2}{c|}{ DADOS DE PESQUISA } & VARIÁVEIS RECODIFICADAS COM VARIÁVEIS DUMMY INTERVALARES \\
\hline $\begin{array}{c}\text { SATISFAÇÃO COM } \\
\text { O ATRIBUTO }\end{array}$ & $\begin{array}{c}\text { SATISFAÇÃO } \\
\text { GERAL }\end{array}$ & PENALIDADES & RECOMPENSAS & $\begin{array}{c}\text { SATISFAÇÃO } \\
\text { GERAL }\end{array}$ \\
\hline 4 & 4 & 0 & 3 & 4 \\
\hline-2 & 3 & -3 & 0 & 3 \\
\hline 1 & 5 & 0 & 0 & 5 \\
\hline
\end{tabular}

\section{Satisfação média}

com o atributo

1

Fonte: Elaborada pelos autores.

Utilizando esse método para o atributo ATıI, já citado nos exemplos anteriores, a equação de regressão fica em $\mathrm{Y}=2$, OI - 0,33Xneg + 0,59Xpos. Nesse caso, a recompensa 0,59 é o acréscimo da satisfação geral para cada unidade 
de acréscimo da satisfação com o atributo quando acima da média. A penalidade 0,33 é o decréscimo da satisfação geral para cada unidade de decréscimo da satisfação com o atributo quando abaixo da média. A vantagem da utilização do valor médio de satisfação atual como referência é que se conhecerá o impacto na satisfação geral para o acréscimo ou decréscimo da satisfação atual com cada atributo. Nesse trabalho, as variáveis dummy foram recodificadas com a referência igual à satisfação média atual com cada atributo. Sendo assim, desloca-se o ponto de alteração do ângulo da reta para onde ocorre o maior número de avaliações e se conhece o impacto na satisfação geral a partir da satisfação média, que é o diagnóstico atual.

\section{ANÁlise do PRC PARA O CASO dOS SUPERMERCADOS}

A análise do PRC pode ser executada pela análise dos coeficientes $\beta$ do modelo multiatributo da satisfação geral com a equação de regressão com variáveis dummy intervalares: $\mathrm{Y}=\beta_{\mathrm{O}}+\beta_{\mathrm{I}} \mathrm{AT}_{\mathrm{I}} \mathrm{pos}+\beta_{2} \mathrm{AT}_{\mathrm{I}}$ neg $+\ldots+\beta_{3 \mathrm{I}} \mathrm{AT}_{\mathrm{I}}$ $\mathrm{pos}$ $+\beta_{32} \mathrm{AT}_{\mathrm{I}} \mathrm{G}^{\text {neg, onde }} A T_{n}$ é a satisfação atual com o atributo $n$. Todos os coeficientes, significantes ou não, poderiam ser considerados valor de penalidade ou recompensa. Entretanto, quanto mais significativo é o coeficiente de regressão, mais seguro é o resultado. Com o pressuposto de que uma organização priorizaria atributos com magnitudes maiores e seguras, pode-se optar em Desconectar atributos com coeficientes insignificantes do modelo de satisfação; dando a oportunidade para que outros atributos que estariam Ocultos no ruído aparecessem como significativos.

Em uma decisão de escolher as variáveis independentes que representem um modelo de satisfação geral mais parcimonioso, o método stepwise pode ser utilizado com o auxílio do software estatístico SPSS. O método stepwise escolhe primeiro a variável independente de maior correlação com a variável dependente. A cada variável independente adicionada no modelo, o programa verifica se alguma variável adicionada anteriormente se tornou insignificante, retirando-a do modelo se necessário (MAROCO, 2003).

Com o método de regressão stepwise, eliminando-se da amostra três casos com resíduos de valores extremos, conseguiu-se o modelo mostrado na Figura 3, que têm um coeficiente de determinação $\mathrm{R}_{\mathrm{a}}^{2}=0,598$, nível satisfatório para ciências sociais segundo Matzler, Sauerwein (2002) e Maroco (2003) e também melhor do que o verificado em Cheung e Lee (2005), Matzler et al. (2004), Fuchs e Weiermair (2004), e Matzler e Sauerwein (2002). A validação dos pressupostos do modelo de regressão, quanto à normalidade dos resíduos, é atendida pelo 
teste de Kolmogorov - Smirnov, com p-value > 0,05. A faixa de VIF (fator de inflação de variância) é menor que 5, não apresentando multicolinearidade que afete o modelo (CHEUNG; LEE, 2005; MAROCO, 2003).

\section{FIGURA 3}

MODELO DA SATISFAÇÃO GERAL

OBTIDO COM REGRESSÃO STEPWISE

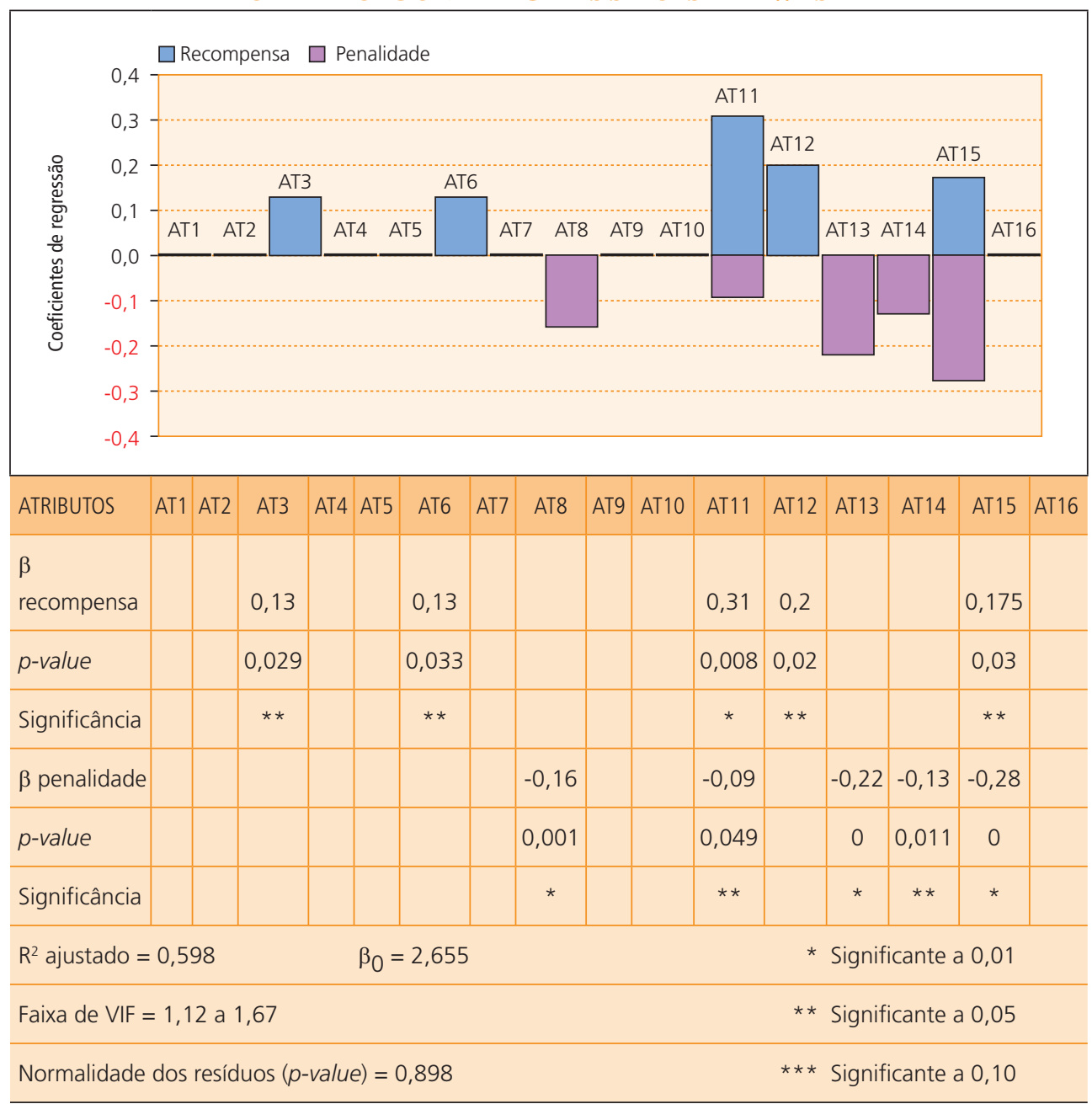

Fonte: Elaborada pelos autores.

Os atributos podem ser classificados em obrigatórios, unidimensionais, atrativos e neutros em função da magnitude dos coeficientes de penalidade e de recompensa (MATZLER; SAUERWEIN, 2002; MATZLER et al., 2004). Os 
atributos obrigatórios, ou básicos, apresentam o coeficiente de penalidade com magnitude significativamente superior ao coeficiente de recompensa. A satisfação geral é influenciada quando a satisfação com o atributo fica abaixo do nível médio de satisfação do mercado, mas não é influenciada quando está acima. Os atributos atrativos, ou excitantes, apresentam o coeficiente de recompensa com magnitude significativamente superior ao coeficiente de penalidade. A satisfação geral é influenciada quando a satisfação com o atributo fica acima do nível médio de satisfação do mercado, mas não é influenciada quando está abaixo. Os atributos unidimensionais, ou de desempenho, apresentam o coeficiente de penalidade com magnitude próxima ao coeficiente recompensa. Nesse caso, a satisfação geral é influenciada pelo nível de satisfação com o atributo quando está acima ou abaixo do nível médio de mercado. Existem ainda os atributos neutros, que não influenciam a satisfação geral, independentemente de seu desempenho. As classificações dos atributos podem também ser obtidas com os coeficientes de regressão plotados no gráfico de dispersão da Figura 4 .

As linhas divisórias dos quadrantes do gráfico da Figura 4 foram estabelecidas pelo ajuste da equação de regressão linear entre os p-value e o módulo dos coeficientes da regressão múltipla de todos os I6 atributos contra a satisfação geral. A equação de ajuste dessa regressão é $Y=-0,0234 \operatorname{Ln}(X)+0,0334$, com $\mathrm{R}^{2}=0,8 \mathrm{I}$. Nesse caso, a variável dependente $(\mathrm{Y})$ é representada pelos coeficientes de regressão e a variável independente $(\mathrm{X})$ é representada pelos $p$-values de cada coeficiente. As linhas divisórias foram estabelecidas em 0,09 , valor que corresponde aos coeficientes com $p$-value igual a o,Io.

\section{FIGURA 4}

CLASSIFICAÇÃO DOS ATRIBUTOS CONFORME A MAGNITUDE

DOS COEFICIENTES DE PENALIDADE E RECOMPENSA

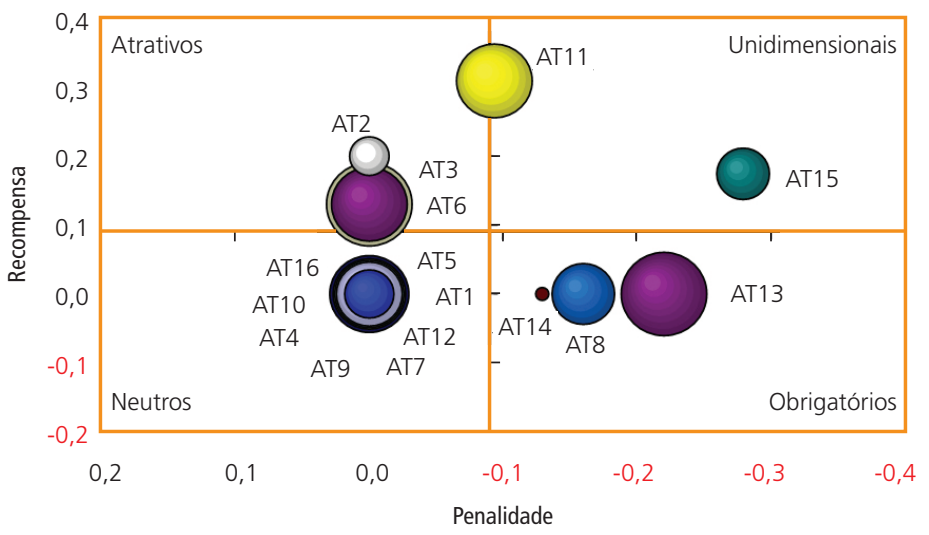

Fonte: Elaborada pelos autores. 
Os níveis de satisfação com os atributos do SMA em relação ao SMB também foram agregados nesse gráfico. Quanto Maior o tamanho do círculo do atributo, Pior a satisfação do SMB em relação ao SMA, seu maior concorrente. O tamanho da circunferência representa prioridade de melhoria para o SMB. Esse recurso é útil quando vários atributos apresentam coordenadas próximas no gráfico e necessita-se definir prioridades de melhoria.

Os atributos ATir Qualidade das frutas, verduras e legumes e ATi5 Cortesia no atendimento podem ser considerados unidimensionais por apresentarem impacto na satisfação geral se a satisfação do atributo aumentar ou diminuir em relação à média do mercado. Os atributos ATı3 Diversidade de marcas por produto, AT8 Limpeza e arrumação na loja e ATı4 Câmeras de vigilância são considerados obrigatórios. Já os atributos AT3 Lojas diversas anexas, AT6 Música ambiente e ATı2 Preço praticado abaixo dos outros supermercados são considerados atrativos.

O nível de satisfação do SMA pode ser comparado com o SMB e com nível médio de satisfação do mercado para definir ações de melhoria. Foi efetuado o teste $t$ para verificar se existe diferença significativa entre os tipos de satisfação a um nível de 90\% de confiança ( $p$-value< o,I) (Tabela 2).

Observando-se os dados da Tabela 3, os consumidores do SMB estão com satisfação inferior aos consumidores do SMA em 9 dos 16 atributos pesquisados e também na satisfação geral. Da mesma maneira, os consumidores do SMB têm uma satisfação geral menor que a média do mercado. Melhorar todos os atributos simultaneamente poderia representar um esforço bastante grande e desnecessário para o SMB. Pela análise do PRC verifica-se que vários atributos não influenciam na satisfação geral de forma significativa, podendo ser ignorados. Para melhorar a satisfação de seus clientes e também sua posição competitiva, o SMB deveria concentrar-se nos atributos atrativos, unidimensionais e obrigatórios. 


\section{TABELA 3}

TESTE T DA DIFERENÇA DA SATISFAÇÃO

ENTRE OS ATRIBUTOS DOS SUPERMERCADOS

\begin{tabular}{|c|c|c|c|c|c|c|c|c|c|c|}
\hline \multirow{2}{*}{ CÓD. } & \multirow{2}{*}{ DESCRIÇÃO } & \multirow{2}{*}{ CLASSIFICAÇÃO } & \multicolumn{4}{|c|}{ SATISFAÇÃO MÉDIA } & \multicolumn{2}{|c|}{ GAP SMA- SMB } & \multicolumn{2}{|c|}{ GAP SMB - MÉDIA } \\
\hline & & & SMA & SMB & OUT SM & MÉDIA & P-VALUE & CONCL. & P-VALUE & CONCL. \\
\hline AT1 & $\begin{array}{l}\text { Proximidade do } \\
\text { supermercado }\end{array}$ & Neutro & 0,99 & 1,14 & 2,04 & 1,50 & 0,76 & Igual & 0,18 & Igual \\
\hline AT2 & Compra por internet & Neutro & 0,91 & $-0,25$ & $-0,47$ & $-0,01$ & 0,00 & Maior & 0,20 & Igual \\
\hline AT3 & Lojas diversas anexas & Atrativo & 1,14 & $-0,21$ & 0,22 & 0,39 & 0,00 & Maior & 0,06 & Menor \\
\hline AT4 & $\begin{array}{l}\text { Local para deixar } \\
\text { crianças }\end{array}$ & Neutro & $-0,22$ & $-1,18$ & $-0,34$ & $-0,52$ & 0,02 & Maior & 0,04 & Menor \\
\hline AT5 & $\begin{array}{l}\text { Degustação de } \\
\text { produtos }\end{array}$ & Neutro & 1,28 & 0,40 & 0,13 & 0,54 & 0,02 & Maior & 0,33 & Igual \\
\hline AT6 & Música ambiente & Atrativo & 1,41 & 0,42 & 0,81 & 0,89 & 0,01 & Maior & 0,09 & Menor \\
\hline AT7 & $\begin{array}{l}\text { Vagas suficientes no } \\
\text { estacionamento }\end{array}$ & Neutro & 1,80 & 1,97 & 1,92 & 1,90 & 0,70 & Igual & 0,41 & Igual \\
\hline AT8 & $\begin{array}{l}\text { Limpeza e arrumação } \\
\text { da loja }\end{array}$ & Obrigatório & 2,80 & 2,07 & 2,34 & 2,41 & 0,05 & Maior & 0,13 & Igual \\
\hline AT9 & $\begin{array}{l}\text { Cartão fidelidade com } \\
\text { bônus progressivo }\end{array}$ & Neutro & 0,55 & $-0,25$ & 0,24 & 0,21 & 0,05 & Maior & 0,09 & Menor \\
\hline AT10 & $\begin{array}{l}\text { Tamanho das filas no } \\
\text { caixa }\end{array}$ & Neutro & 0,89 & 0,51 & 1,19 & 0,93 & 0,35 & Igual & 0,10 & Igual \\
\hline AT11 & $\begin{array}{l}\text { Qualidade das frutas, } \\
\text { verduras e legumes }\end{array}$ & $\begin{array}{l}\text { Unidimen- } \\
\text { sional }\end{array}$ & 2,64 & 1,59 & 1,89 & 2,03 & 0,01 & Maior & 0,08 & Igual \\
\hline AT12 & $\begin{array}{l}\text { Preços abaixo da } \\
\text { média do mercado }\end{array}$ & Atrativo & 1,62 & 1,85 & 0,88 & 1,35 & 0,53 & Igual & 0,05 & Maior \\
\hline AT13 & $\begin{array}{l}\text { Diversidade de marcas } \\
\text { por produto }\end{array}$ & Obrigatório & 2,61 & 1,32 & 1,60 & 1,83 & 0,00 & Maior & 0,06 & Menor \\
\hline AT14 & Câmeras de vigilância & Obrigatório & 1,01 & 0,97 & 0,26 & 0,66 & 0,91 & Igual & 0,16 & Igual \\
\hline AT15 & $\begin{array}{l}\text { Cortesia no } \\
\text { atendimento }\end{array}$ & $\begin{array}{l}\text { Unidimen- } \\
\text { sional }\end{array}$ & 1,94 & 1,45 & 1,88 & 1,79 & 0,15 & Igual & 0,12 & Igual \\
\hline AT16 & $\begin{array}{l}\text { Disponibilidade } \\
\text { de sanitários para } \\
\text { clientes }\end{array}$ & Neutro & 1,15 & 0,70 & 0,31 & 0,66 & 0,31 & Igual & 0,45 & Igual \\
\hline & Geral & & 2,64 & 1,90 & 2,22 & 2,26 & 0,03 & Maior & 0,09 & Menor \\
\hline
\end{tabular}


Iniciando pelos atributos obrigatórios, uma análise da Tabela 3 mostra que o SMA está com desempenho superior ao SMB nos atributos AT8 Limpeza e arrumação da loja e ATiz Diversidade de marcas por produto. Nesse caso, é urgente que o SMB melhore esses itens, pelo menos até o nível da média do mercado. Quanto aos atributos unidimensionais, o SMB está perdendo para o SMA no atributo ATiı Qualidade das frutas, verduras e legumes e empatando no atributo ATI5 Cortesia no atendimento. Como esses atributos influenciam a satisfação geral de forma significativa se tiverem desempenho acima da média do mercado, o SMB poderia priorizá-los em suas ações de melhoria. O SMA está com satisfação acima do SMB em quase todos os atributos atrativos, o que pode justificar a razão de seus clientes terem satisfação geral superior. O SMB poderia escolher alguns desses atributos para melhoria e aumentar a satisfação de seus clientes. Pela Figura 3 anterior, verifica-se que o atributo de maior impacto seria o ATi2 Preços abaixo da média do mercado. Contudo, o atributo AT6 Música ambiente poderia ser de menor custo para a implantação, uma vez que a satisfação dos clientes do SMB com os preços já está acima da média do mercado. Os demais atributos, considerados neutros, não influenciam a satisfação geral e podem ser ignorados.

\section{LIMITAÇõES DO PRC}

Apesar de identificar como os diversos atributos impactam na satisfação geral, o PRC tem algumas limitações. A primeira delas é que o método tende a não identificar atributos inovadores. De modo geral, atributos inovadores apresentam baixo coeficiente de regressão porque quando o respondente não tem experiência com o atributo, tende a avaliar seu nível de satisfação como neutro ou negativo, como visto no primeiro gráfico da Figura 5, à esquerda. O gráfico mostra a correlação entre as avaliações neutras e negativas de satisfação e a taxa de percepção da presença dos atributos, com $\mathrm{R}^{2}$ de 0,89 . Quanto menor a taxa de percepção do atributo, mais ocorreram avaliações neutras e negativas, indicando baixa influência na satisfação geral. O segundo gráfico apresenta a correlação entre a importância dos atributos derivada estatisticamente e a taxa de percepção deles. Com R R de o,6I (se não fosse o outlier ATı6 indicado por uma seta, ter-se-ia $\mathrm{R}^{2}$ de 0,89 ), há a tendência de que atributos com baixa percepção pelos consumidores apresentem baixo coeficiente de regressão. O terceiro gráfico apresentou $\mathrm{R}^{2}$ de O,I4 para a correlação entre a taxa de percepção dos atributos e os coeficientes de regressão da equação de regressão com variáveis dummy (penalidade e recompensa). Esse valor de $\mathrm{R}^{2}$ é considerado baixo, contudo não invalida o que visualmente se percebe: atributos com baixo nível de percepção têm baixo coefi- 
ciente de regressão dummy e atributos com alto nível de percepção, alto ou baixo coeficiente. Isso ocorreu porque atributos com alta percepção não apresentam necessariamente alto coeficiente de regressão, pois podem existir atributos que não são novos, mas não afetam a satisfação geral.

\section{FIGURA 5}

\section{CORRELAÇÃO ENTRE TAXA DE PERCEPÇÃO DOS} ATRIBUTOS E AVALIAÇÕES DE SATISFAÇÃO
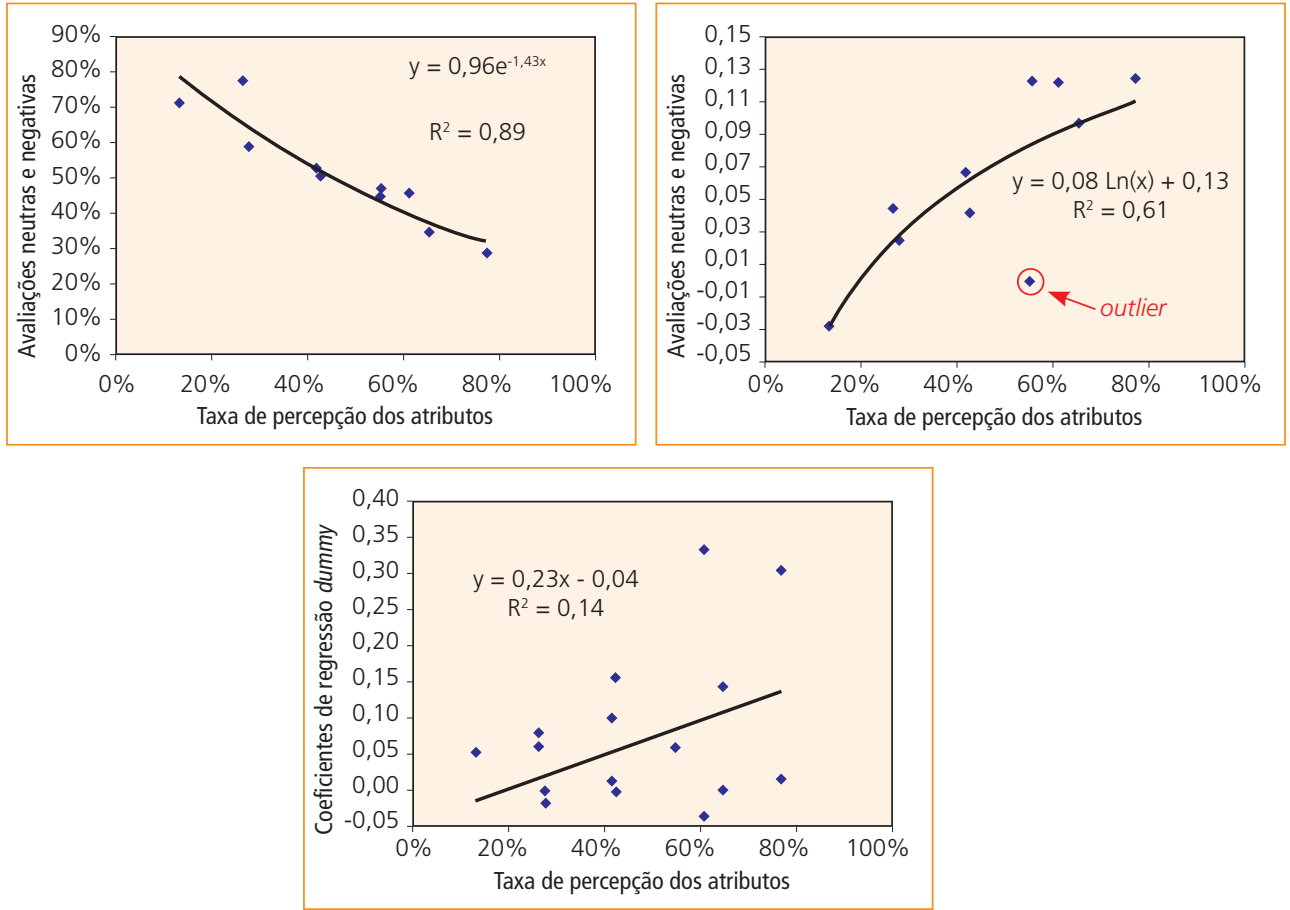

Fonte: Dados da pesquisa.

Dessa forma, a análise do PRC tende a indicar como não significativos os atributos inovadores porque o cliente não tem experiência com eles. Porém, o PRC apresenta a vantagem de que se a organização quiser focar apenas nos atributos conhecidos pelos clientes, poderá priorizar investimentos de melhoria naqueles que mais causam impacto na satisfação geral.

Outra limitação diz respeito à sensibilidade do PRC quanto à divisão da escala para recodificação das variáveis de recompensa e de penalidade. No caso dos supermercados, apresentado neste artigo, se a divisão da escala for feita pelo centro da escala (zero na escala de $-5 \mathrm{a}+5$ ) em vez de ser realizada pelo valor da média da satisfação com os atributos, os resultados serão diferentes. $\mathrm{O}$ atributo 
AT3 passa de atrativo para neutro. $\mathrm{O}$ atributo ATII passa de unidimensional para atrativo e o atributo ATiz passa de obrigatório para unidimensional. A posição do valor de divisão da escala é uma decisão crítica a ser avaliada na fase de projeto da pesquisa. Fazer a análise do PRC com algumas alternativas para a divisão da escala pode ajudar na definição das estratégias de melhoria do produto ou serviço sob investigação.

\section{CONSIDERAÇÕES FINAIS}

Consumidores satisfeitos tendem a repetir o consumo e a divulgar a organização para outros consumidores em potencial, aumentando possivelmente o volume de negócios e o lucro operacional da empresa. Logo, conhecer os atributos que apresentam o melhor potencial de influência na satisfação geral e verificar seu desempenho competitivo é questão-chave para as organizações (ANDERSON; MITTAL, 2000).

A análise por meio da matriz de importância e desempenho tem sido amplamente utilizada para a identificação dos atributos que deveriam ter seu desempenho aumentado para melhorar a posição competitiva de bens e serviços. Porém, a matriz de importância e desempenho considera que importância é uma dimensão independente de desempenho e que a relação entre desempenho dos atributos e satisfação geral do consumidor é linear. Esses pressupostos têm sido criticados em vários trabalhos publicados. De maneira geral, esses estudos têm afirmado que a importância aumenta com o desempenho dos atributos e que a relação entre desempenho e satisfação geral é não-linear. O modelo Kano de qualidade identifica a relação não-linear entre desempenho dos atributos e satisfação geral, contudo não permite priorizar os atributos que devem ser melhorados tendo em vista a posição competitiva do produto analisado.

Este artigo apresentou a análise do contraste da penalidade e da recompensa (PRC) como uma alternativa à matriz de importância e desempenho e ao modelo Kano de qualidade, para a verificação de pontos críticos de melhoria e de um serviço. A análise do PRC, obtida por uma equação de regressão com variáveis dummy, comprovou a relação não-linear entre satisfação com os atributos de supermercados e satisfação geral. O método demonstra quais atributos influenciam na satisfação geral caso fique abaixo da média do mercado, acima da média do mercado ou em ambas as situações, desde que o consumidor tenha experiência com os atributos avaliados. Isto é, atributos inovadores tendem a ser considerados não significativos por esta técnica.

Na pesquisa realizada neste trabalho há dois grupos de atributos. Um relacionado à estrutura dos supermercados, dito dicotômico, pois seu desempenho 
pode ser medido pela presença ou ausência, e outro que depende de procedimentos de operação (AT8, ATıo, ATıI, ATı2, ATı3 e ATı5). O único atributo desse último grupo que não apresenta interferência na satisfação geral é o ATıo (Tamanho das filas no caixa). Dos atributos do primeiro grupo, apenas o AT3 e AT6, com magnitudes de seus coeficientes menores que os atributos do segundo grupo, mostraram interferência na satisfação geral. Esses resultados podem indicar que a satisfação geral seja mais dependente de atributos relacionados a procedimentos de operação do que à estrutura física. Novas pesquisas devem ser desenvolvidas para confirmar tal indicação.

\section{REFERÊNCIAS}

ANDERSON, E. W.; MITTAL, V. Strengthening the satisfaction-profit chain. Journal of Service Research, Maryland, v. 3, n. 2, p. I07-I20, Nov. 2000.

AUDRAIN, A. F. A study of the attributes - overall satisfaction links for a service. In: CONFÉRENCE DE L'AUSTRALIAN AND NEW ZEALAND MARKETING ACADEMY (ANZMAC), Wellington, Nouvelle-Zélande, nov. 2004. Disponível em: <http://130.195.95.71:808I/www/anzmac2004/ cdsite/papers/audraini.pdf>. Acesso em: 22 abr. 2005.

BERGER, C. et al. Kano's methods for understanding customer-defined quality. Journal of the Japanese Society for Quality Control, Tokyo, v. 23, n. 2, p. 3-35, I993.

BRANDT, R. D. A procedure for identifying value-enhancing service components using customer satisfaction survey data. In: SURPRENANT, C. (Ed.). Add value to your service. Chicago: American Marketing Association, I987. p. 6I-65.

CHEUNG, C. M. K.; LEE, M. K. O. The asymmetric effect of website attribute performance on satisfaction: an empirical study. In: $38 \mathrm{TH}$ HAWAII INTERNATIONAL CONFERENCE ON SYSTEM SCIENCES. 2005. Disponível em: <http://csdl2.computer.org/comp/proceedings/hicss/ 2005/2268/07/22680I75c.pdf>. Acesso em: 9 jun. 2005.

FUCHS, M.; WEIERMAIR, K. Destination benchmarking: an indicator system's potential for exploring guest satisfaction. Journal of travel research, Boise, v. 42, p. 2I2-225, Feb. 2004.

GARVER, M. S. Best practices in identifying customer-driven improvement opportunities. Industrial Marketing Management, Vernon, v. 32, p. 455-466, 2003.

HUISKONEN, J.; PIRTTILÄ, T. Sharpening logistics customer service strategy planning by applying Kano's quality element classification. International Journal on Economics, Amsterdam, v. 56-57, p. 253-260, 20 Sept. 1998.

JURAN, J. M. A qualidade desde o projeto: novos passos para o planejamento da qualidade em produtos e serviços. 2. ed. São Paulo: Pioneira, I992.

KANO, N. et al. Attractive quality and must-be quality. Journal of the Japanese Society for Quality Control, Tokyo, p. 39-48, Apr. I984.

KOTLER, P. Administração de marketing: a edição do novo milênio. ıo. ed. São Paulo: Prentice Hall, 2000. 
MAROCO, J. Análise estatística: com utilização do SPSS. Machester: Edições Silabo, 2003. MARTILLA, J. A.; JAMES, J. C. Importance performance analyses. Journal of Marketing, Chicago, n. 9, p. 4I-77, I977.

MATZLER, K. et al. How to delight your customers. Journal of Product \& Brand Management, Santa Barbara, v. 5, n. 2, p. 6-I7, I996.

. The asymmetric relationship between attribute-level performance and overall customer satisfaction: a reconsideration of the importance-performance analysis. Industrial Marketing Management, Vernon, v. 33, n. 4, p. 27I-277, 2004.

MATZLER, K.; SAUERWEIN, E. The factor structure of customer satisfaction. International Journal of Service Industry Management, Karlstad, v. I3, n. 4, p. 3I4-332, 2002.

MITTAL, V.; ROSS, J.; William, T.; BALDASARE, P. M. The asymmetric impact of negative and positive attribute-level performance on overall satisfaction and repurchase intentions. Journal of Marketing, Chicago, v. 62, p. 33-47, Jan. I998.

PICOLO, J. D. Influência do desempenho de atributos de produtos ou serviços na satisfação dos clientes: uma análise comparativa entre diferentes técnicas de pesquisa. 2005. I97 f. Dissertação (Mestrado em Administração)-Centro de Ciências Sociais Aplicadas, Universidade Regional de Blumenau, Blumenau, 2005 .

TING S. C.; CHEN, C. N. The asymmetrical and non-linear effects of store quality attributes on customer satisfaction. Total Quality Management, Abingdon, v. I3, n. I4, p. 547-569, 2002.

TONTINI, G.; SILVEIRA, A. Identification of critical attributes of success in products and services: an alternative to importance - performance analysis. In: 2005 BALAS ANNUAL CONFERENCE, 2005, Madrid. Proceedings... Madrid: Instituto de Empresa, 2005. p. I-I5.

\section{TRAMITAÇÃO}

Recebido em 14/12/2006

Aprovado em 16/7/2008 